\title{
New Studies of Thermal Evolution Process
}

\author{
Alexandre Titov
}

\begin{abstract}
Using new experimental techniques of synchronous differential thermal measurements, studies of thermal evolution processes are performed in a tungsten carbide gauge block that is, in the material often used in interferometric length measurements in international comparisons. It is shown experimentally that the consecutive states of the system under our experimental conditions can be only described as an irreversible in time, non-repeatable evolution process that is characterized by braking symmetries in time and in space. As the studied system is an open one, the standard laws of conservation of energy and momentum for the system (following from the theories developed in the framework of Newtonian paradigm) are clearly violated. The experiments, performed with the new material, obviously demonstrate that the superposition principle is not valid for electromagnetic fields interacting with material objects. It is proved that the fluxes of energy and momentum of the external field through the surface of the material object is the driving source of all changes in the properties and in the structure of the irradiated object. The hysteresis curves, which are changing continuously with the variations of the observation position and in time, characterize the evolution process that is observed in two different locations on the surface of material artifact but at the same time moment. All the changes inside the object are associated with the propagation of the field-particle system within the material artifact as a response to the absorption of the fluxes of energy and momentum of the external field that is created by all external partners of the specified object in the system. The parameters of the propagation of the field-particle system (velocity, amplitude) depend critically on the previous history of the object. The interaction of material objects through the common field realizes the Leibniz's principle of "interrelation of one to all, and of all to one". When the evolution of each material object occurs under the influence of the infinite number of correlated factors, the description of the resulting non-repeatable, irreversible in time process is not possible in terms of mathematics. Here, we present one of the experimental demonstrations of the basic principle of Leibniz relational world:" Every simple Substance is by its nature a concentration, and a living mirror of the whole Universe, according to its Point of view".
\end{abstract}

Index Terms - hysteresis effect, non-repeatable evolution process, superposition principle invalidity.

\section{INTRODUCTION}

We present here further experimental investigations of the thermal evolution process $[1,2]$, in which an initially homogeneous material artifact (as a result of the act of absorption of some portions of energy and momentum of external electromagnetic (EM) field) starts to change significantly its physical properties. These changes occur due

Alexandre Titov, Physics Department, Yeditepe university, Istanbul, Turkey. antitov243@gmail.com to the propagation of the field-particle system $[3,2]$ inside the material object. All our experiments are well beyond the description of theoretical physics, as a simple consequence of the basic limitations in finding the mathematic solution even to a "relatively simple" three-body problem of H. Poincaré. So, these studies are significant in many fields and applications. For example, they are crucial for the test of the validity of contemporary physical theories [4]; for technical progress in the areas where heat and mass transfer is of primary importance; for metrology (especially, in the fields of precise length and temperature measurements). All the experiments presented here lead to the conclusion that the interactions between material objects in the Universe through the EM field result in time irreversible, non-repeatable evolution processes in each of the material objects. This type of process (having an enormous number of interrelated influence factors) cannot be in principle adequately described in the language of mathematics, in strict agreement with the fundamental Kurt Gödel's incompleteness theorem [5] of the year 1931.

\section{EXPERIMENTAL SETUP AND MEASUREMENT METHOD}

It is known from electrodynamics $[6,3]$ that the act of absorption of the energy and momentum of an external electromagnetic (EM) field (that is irradiating the surface of a material artifact) results in the increase of the energy of the field-particle system inside the artifact [6]. The act of absorption is followed by the propagation of the fluxes of energy and momentum of the field-particle system inside the object [3]. For experimental studies of this type, the most appropriate is the method of synchronous differential thermal measurements $[7,8]$. The block-diagram of our experiment is shown in Fig. 1.

A 100-mm tungsten carbide (TC) gauge block (GB), often used in many international comparisons as a length standard, was located at the bottom of a closed Dewar and was supported by three steel $5 \mathrm{~mm}$ spheres inserted into a thick porous material. An air conditioning system realized the long-term temperature stabilization in a selected region of the laboratory with typical diurnal temperature variations of about $50 \mathrm{mK}$. As it follows from Fig. 1, on the larger side surface of the GB (with the width of $35 \mathrm{~mm}$ and the height of $9 \mathrm{~mm}$ ) were installed three thermometers in copper adapters. In the center of this measuring system, a $100-\mathrm{Ohm}$ platinum resistance thermometer (PRT) was located symmetrically relative to two thermistors. The thermistors, used as thermometers in measuring channels 1 and 2, had a resistance value of about 18 kilo-Ohms (at room temperature), and their thermal sensitivities were quite close. The side surfaces of the adapters of the thermometers were located parallel to the gauging 
surfaces of the GB (Fig. 1). The distances between the surfaces of the adapters of the PRT and thermistors were 10 or 13.5 $\mathrm{mm}$. The adapters covered the whole width of the block, so that the system was designed to measure the temperature variations only in one direction, along the longer axis of the GB.

The PRT was connected to a programmed, high-precision DC bridge Mi-T615 (Measurement International, Canada). The records of the variations of the thermistor resistances as functions of $\mathrm{GB}$ temperatures at the locations of the thermistors were realized by precision multi-meters HP-3450A. These multi-meters (with improved time stability of the base) were used in a special regime: half of the measuring period the instrument was measuring the signal, and the other half-period it was measuring the electronic offsets. The measured offsets were then used to correct automatically the measuring results. Besides that, as the used measuring current in the thermistors was only $50 \mu \mathrm{A}$, the calibration of each thermistor was performed together with the Hp multi-meter of the same channel. Small drifts of the measuring current of the multi-meter were possible to correct by regular measurements of the resistance of a temperature stabilized standard resistor, whose value was selected close to the thermistor values. The power dissipated by the thermistor was only $\sim 22.5 \mu \mathrm{W}$, and it could not change significantly the results of the studies.

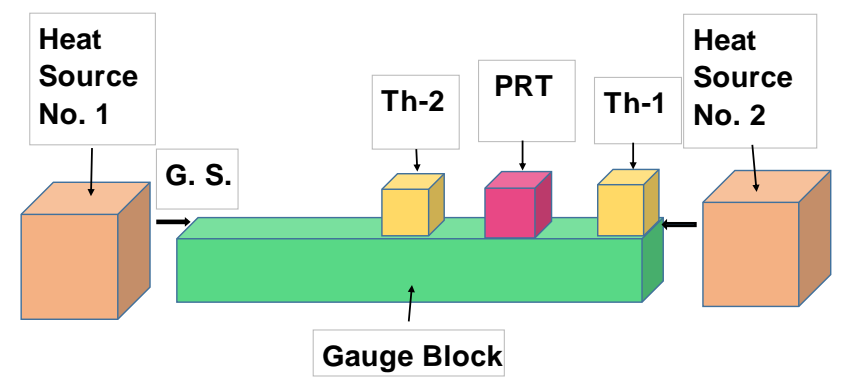

Fig.1. Part of experimental setup located inside a Dewar: TC gauge block, with two gauging surfaces (G.S) indicated by arrows; platinum resistance thermometer (PRT) in copper adapter; two thermistors belonging to measurement channels 1 and 2; auxiliary heat sources to produce a desired energy flux inside the block.

The use of the adapters gave the possibility to reduce drastically the temperature gradients in the contacts between the surfaces of the GB and the adapters, so that it opened the opportunity to realize the calibration of the self-heating of the thermometers on the surface of a particular material with a few $\mu \mathrm{K}$ uncertainty $[9,10]$. In our experiments, the calibration of the measuring channels 1 and 2 was realized inside the Kōsters interferometer equipped with its own temperature stabilizing system, so that temperature gradients in the gauge block (on whose surface the calibrations were performed) were an order of magnitude smaller than in case of our Dewar system. In this way, all the necessary requirements for high precision temperature measurements were fulfilled [9].

These calibrations were realized using a precision Rosemount (USA) standard 25-Ohm platinum resistance thermometer (SPRT), which was also equipped by a special copper adapter that was in contact with the GB surface. We used the Mi-bridge, which was programmed to change the current in the PRT), and used the procedure of calibration described in $[9,10]$. As a result, we had two thermistor channels that were able to measure the temperature difference of the GB surface in the close vicinity of the adapters of our thermistors with a few $\mu \mathrm{K}$ uncertainty.

We also used a newly developed method of synchronous differential temperature measurements (SDTM) [7, 8] that gave the opportunity to detect and to measure with high precision the simultaneous variations of temperatures in the thermistors channels that were induced by the periodic modulation of the current in the PRT. Synchronous records of the resistances in the channels (Fig. 2), together with the calculations of the corresponding values of temperature and of thermal velocity in each channel were performed by our special program. These calculations were based on the stored calibration equations for all thermometers that were in advance loaded into the program.

Program's print-screen for 3 periods of the modulation cycle is presented in Fig. 2. The duration of one cycle was $\sim 2.5$ hours. During the first 37 minutes of the modulation cycle, the PRT current was kept at the level of $5 \mathrm{~mA}$ (heating period), and for the cooling period (the last 111 minutes) the current was equal to $1 \mathrm{~mA}$. As the current modulations were performed by the high-precision DC bridge, the accurate temperature measurements of all three thermometers were available during the whole modulation cycle.

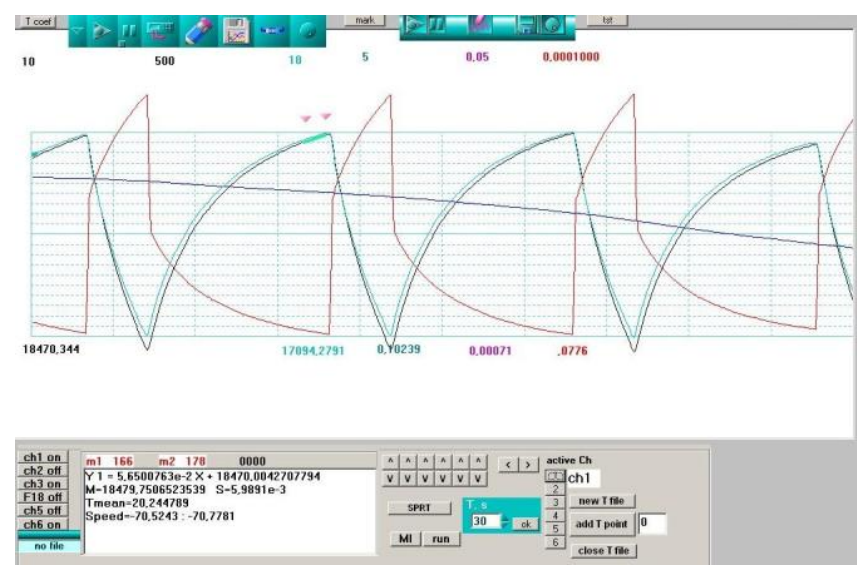

Fig.2. Simultaneous records of the variations of the resistances: of the PRT (red line); of the two thermistors Th-1 and Th-2 (black and green lines, respectively); of special thermistor-3 measuring the temperature in the laboratory (blue line, corresponding to the air temperature variations of about $35 \mathrm{mK}$ during the time interval of 500 minutes). The heating period of the modulation current is three times shorter than that of the cooling period of the cycle. Two cursors (shown by pink triangles) define the specified time interval, for which the program calculates the mean values of the artifact temperature and of the temperature velocity in the selected channel. The corresponding values are presented in the special window, together with the corresponding time interval. (See text for other details).

The red record in Fig. 2 (with a faster time response) corresponds to the measured variations of the PRT resistance. The two other traces (black and green) show the variations of the resistances of the thermistors characterized by negative thermal coefficients. The blue line corresponds to the air temperature variations in the laboratory with the total spread of a few tens of $\mathrm{mK}$. The two pink triangle cursors specify the time interval in 
minutes for which the program, using the stored calibration equations calculates and presents in the window the mean temperature and the thermal velocity values for the specified time interval and for the selected channel. As a consequence of the used setup, the system is extremely sensitive to any asymmetry in heat fluxes along the surface of the GB in the direction of the longest side of the GB. The key features of the experiment are that both thermistor channels are calibrated to measure the corresponding temperature of the artifact surface in the close vicinity of the thermometer adapter and that the collected by the program temperature information refers to the same time instant.

\section{RESULTS AND DISCUSSIONS}

When all the time intervals are specified for each modulation cycle and the recorded variations of the resistance values in all three channels are converted into the temperature information, then it is possible to process further this information. For example, the resistance information of Fig. 2 can be converted into the thermal velocity information, which can be shown as a time dependence of the vector quantity $\mathbf{V}[\mathbf{1 , 2}]$ in Fig. 3. Here, the quantity $\mathbf{V}[\mathbf{1 , 2}]$ is defined as a difference between the thermal velocities $(\mathbf{V}[\mathbf{1}]-\mathbf{V}[\mathbf{2}])$ recorded in the channels 1 and 2 , respectively. The quantity $\mathbf{V}[\mathbf{1 , 2}]$ is a vector, and it explicitly contains the direction that is specified by the locations of thermistors 1 and 2 . It is necessary to emphasize here, that the data points of Figs. 2 and 3 correspond to a special experiment, when the whole measurement system was moved along the axis of the GB, so that one of the side surfaces of the adapter of the thermistor 2 was in the plane of the other (left) gauging surface of the block. This configuration was chosen opposite to the standard position of Fig. 1 (that was used in the studies $[1,7]$ ) in order to demonstrate the effect of the position of the PRT (and thus the energy absorption area on the gauge block surface) on the manifestations of the evolution process in the artifact.

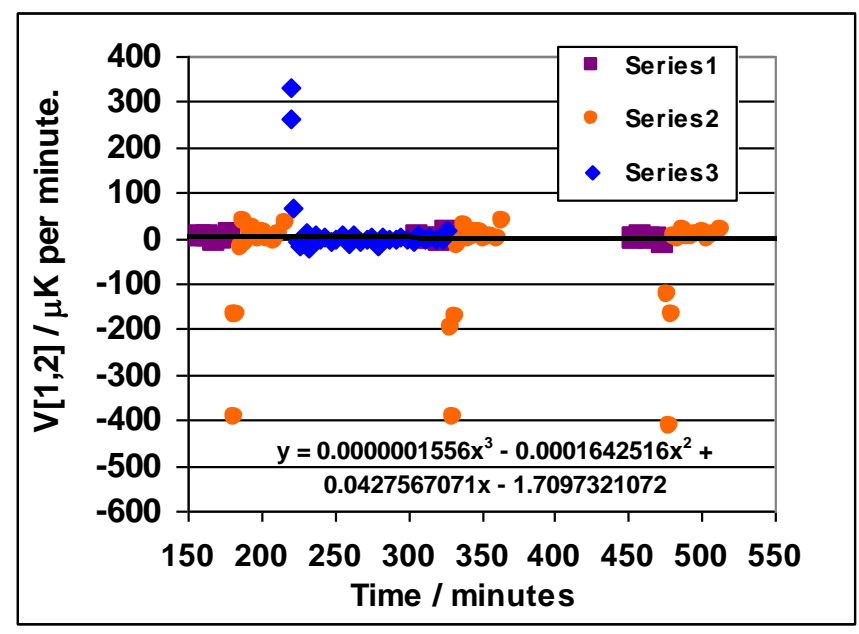

Fig.3. The dependence of the difference in temperature velocities $\mathbf{V}[\mathbf{1 , 2}]$ in the channels 1 and 2 as function of time. The data points for the heating period are shown as red dots, and for the cooling period as blue rhombi. The reference points, to which the polynomial fit is calculated, are shown as squares. The equation of the fit is presented in the inset. The solid line on the plot shows the reference function, relative to which the quantity $\Delta \mathbf{V}[\mathbf{1 , 2}]$ is measured.
In Fig. 3, red dots correspond to the data points of the heating period of the modulation cycle, and blue rhombi represent the data points of the cooling period of the cycle. The last 25 minutes of the cooling period contain our reference points, which in Fig. 3 are shown as squares and which correspond to 5 minutes averaging time. A third order polynomial fit to all reference points is shown in the figure as a solid line, and its equation (defining the reference function) is presented in the inset. The reference function carries the information about the effect of long-term variations of the external temperature on the quantity $\mathbf{V}[\mathbf{1 , 2}]$, as well as the effect of the possible long-term drifts of the thermistor sensitivities. These effects can be, practically, cancelled out by the computer, when determining the value of each data point relative to the reference function. As a result of the fact that the cooling period is three times longer than the heating period of the modulation cycle, we find that the difference between the thermal velocities $\mathbf{V}[\mathbf{1}]$ and $\mathbf{V}[2]$ is becoming quite negligible at the end of modulation cycle, when we average the difference over several cycles. This follows from the fact that the mean difference between all the reference points and the reference function in Fig. 3 is less than $0.4 \mu \mathrm{K} /$ minute. Meanwhile, the perturbations of the studied system by external random factors for the reference points are described by the standard deviation of $6 \mu \mathrm{K} /$ minute, which characterize the spread of a single measurement in the series. But it is possible to realize the measurements of the quantity $\mathbf{V}[\mathbf{1 , 2}]$ relative to the reference function, denoting it by $\Delta \mathbf{V}[\mathbf{1 , 2}]$. For this vector quantity it is possible to perform averaging over several modulation cycles and realize the basic property of the synchronous detection: the systematic effect that is induced by the modulation will be accumulated, while the contribution of random perturbations will be systematically reduced $[1,2,8]$. The corresponding plot for the quantity $\Delta \mathbf{V}[\mathbf{1 , 2}]$ is presented in Fig. 4, where averaging is performed over all 3 cycles of Fig. 3. The improvement of signal-to-noise ratio relative to Fig. 3 is quite evident.

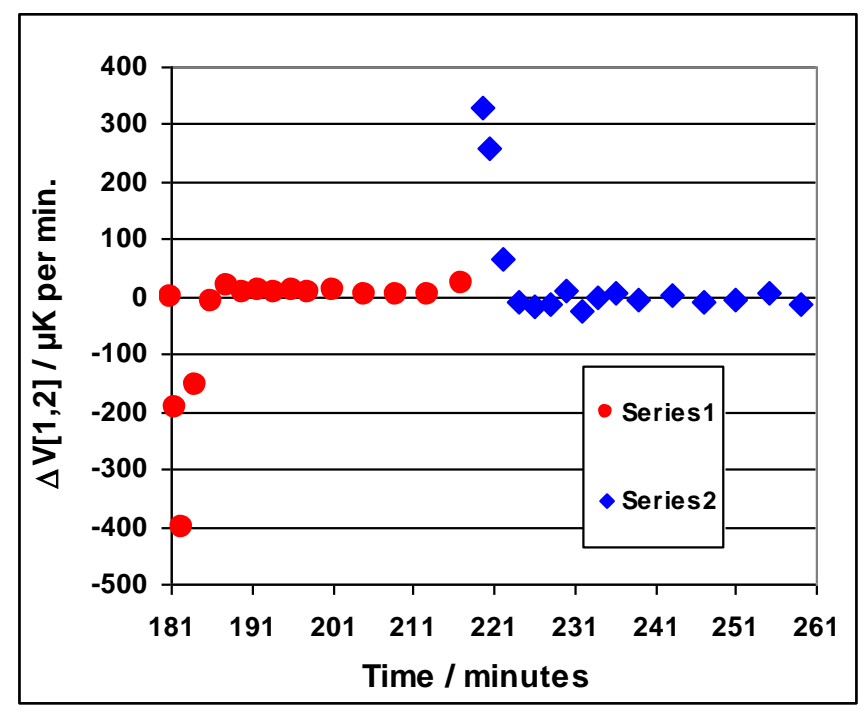

Fig.4. The dependences of the quantity $\Delta \mathbf{V}[\mathbf{1 , 2}]$ as a function of time averaged over several modulation cycles (presented in Fig. 2).

The distance between the adapters $(\Delta \mathrm{X})$ was $10 \mathrm{~mm}$.

Naturally, the difference in the induced thermal velocities in the thermistor channels 1 and 2, which is represented by 
$\Delta \mathbf{V}[\mathbf{1 , 2}]$, is also a vector quantity. For example, the positive value of $\Delta \mathbf{V}[\mathbf{1 , 2}]$ corresponds to the case when the resulting flux of energy of the field-particle system to the unit volumes of the artifact in the vicinity of thermistor 1 is larger than the corresponding flux to the unit volumes in the vicinity of thermistor $2[2,8]$.

When comparing the plots of Fig. 4 here with the plot of Fig. 5 in [1], we immediately realize that the sign of the effects is opposite. Indeed, the sharp peak of the quantity $\Delta V[1,2]$ at the beginning of the heating period in Fig. 4 here indicates that flux of energy was larger in the direction of the location of the thermistor-2, while in the plots of Fig. 5 in [1] and Fig. 3 in [2] the flux was larger in the direction of the thermistor- 1 . This observation agrees with the conclusion in $[1,2]$ that the larger flux of energy at the beginning of the heating period occurs in the direction of the thermistor that is closer to the gauging surface of the block. Also, in the agreement with the experiments of [1,2], another sharp peak of the opposite sign in the differential energy flux occurs at the beginning of the cooling period of the modulation cycle. In Fig. 4 this peak is presented by the data points (shown as blue rhombi) for the time interval of $0.5-3$ minutes of the cooling period.

There are also important differences between the results of experiments performed on the steel $[1,2,7]$ and tungsten carbide blocks presented here. First, in TC block the maximum value of the energy peak was about $25 \%$ smaller than that in a steel artifact. Second, the evolution processes in steel and TC blocks have quite different time scales. For example, in the experiments when the thermistor center was shifted by 14.5 $\mathrm{mm}$ relative to the nearest gauging surface, the peak value of the quantity $\Delta \mathbf{V}[\mathbf{1 , 2}]$ was observed at the time interval of about 1.58 minutes of the heating period for TC block, and only at 3.52 minutes for the steel GB [2]. Third, the value of the quantity $\mathbf{\Delta V}[\mathbf{1 , 2}]$ in Fig. 4 is negative during the time interval from the very beginning up to 6 minutes of the heating period. Then, from the sixth minute it is becoming positive (but with relatively small amplitude) and keeps the positive sign up to the end of the heating period. In the steel 100-mm GB for the same positions of thermometers the quantity $\Delta \mathbf{V}[\mathbf{1 , 2}]$ keeps the sign during the heating period. Naturally, the different behavior of the energy fluxes in time in different materials will result in the different shapes of the hysteresis curves, which characterize the evolution process in different parts of the material artifact, but for the same time instant.

The change of the direction of the flux of the field-particle system in the material with absorption means the change of the directions of the resultant force acting on the unit volumes inside the material [3,2]. This change of the net force (acting on two unit volumes) is accompanied by inevitable changes in stresses and deformations inside the initially homogeneous material, and it also results in some mass and charge transfer $[2,3]$ to counterbalance the external perturbations. Using the analogy with ferro-electric materials, hysteresis curves can be used to characterize the thermal evolution processes under different experimental conditions $[2,11]$. The hysteresis curve, corresponding to the experimental data presented in Fig. 4, is shown in Fig. 5. Here, as in [1, 2], the variable in $\mathrm{y}$-axis is the temperature difference $\Delta \mathbf{T}[\mathbf{1 , 2}]$ between the channels 1 and 2 that is induced by the variations of the dissipated power in the PRT. The heating period of the modulation cycle in this figure is presented again by red dots and covers the time interval marked by the arrows A and B. The time direction in this part of the plot corresponds to the case when the excessive force is applied to the unit volumes of the artifact in the vicinity of the thermistor-2. The cooling period of the cycle is presented by blue rhombi and covers the time interval from B to $\mathrm{C}$ that is measured in the opposite direction. This procedure corresponds to the opposite direction of the excessive force that is applied to the unit vectors in the vicinity of the thermistor-1. Naturally, the corresponding dependence $\Delta T[1,2]$ can be presented as a sweep over the continuous arrow of time, similar to the dependence shown in Fig. 4. But the hysteresis curve presentation is easier for eye estimation, and it is traditional in the hysteresis effect studies since the first publication in the field by J. A. Ewing [12] in 1882. The information about the time interval durations is kept through the whole curve of Fig. 5, and it is immediately clear from the plot that the duration of the cooling period is three times longer than that of a heating period. It is also necessary to note here, that the difference in the $y$-values of the data points marked by $\mathrm{A}$ and $\mathrm{C}$ is, practically, zero. This difference cannot be observed in the scale of the presented figure, as the data points for the whole modulation cycle in Fig. 5 are determined relative to the corresponding reference function [1, 2], and the random fluctuations for the points $\mathrm{A}$ and $\mathrm{C}$ are approaching to zero with the increase of the number of the used modulation cycles.

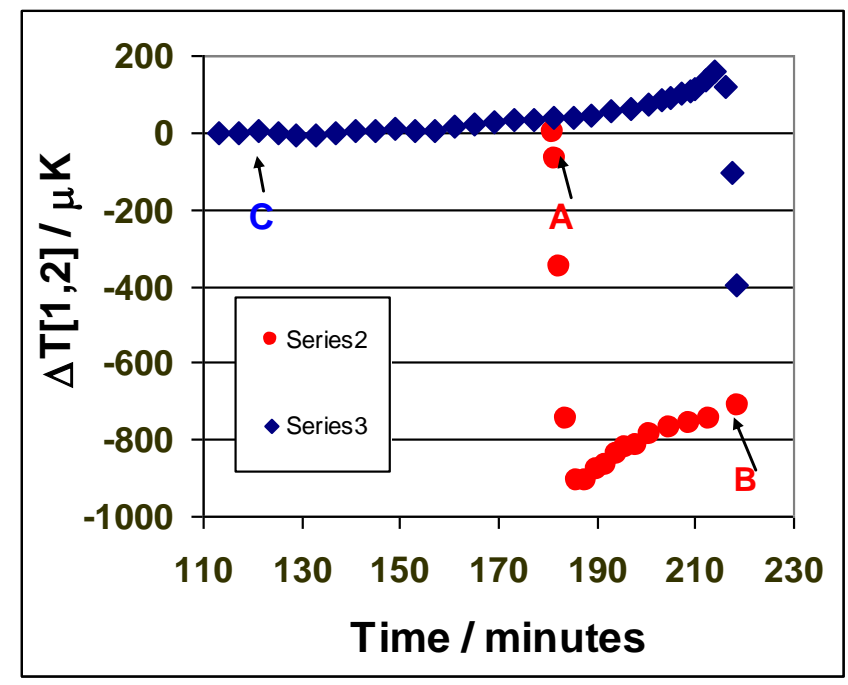

Fig.5. The record of the thermal hysteresis curve, when thermistor-2 was close to the gauging surface of the block, the temperature difference $\mathbf{T}[\mathbf{2 , 1}$ ] was $0.88 \mathrm{mK}$, and the distance between the adapters was $10 \mathrm{~mm}$.

It is worth emphasizing here, that shapes of the hysteresis curves in tungsten carbide and in steel GB are quite different (see, for example, Fig. 6 in [1]). For TC block the hysteresis curve passes through an extreme value during the heating period of the cycle. This is the direct consequence of the fact that the energy flux in Fig. 4 is changing the direction already during the heating period of the modulation cycle. The other significant difference is in the values of the hysteresis effects in the TC and steel gauge blocks for the observations performed under identical experimental conditions. For example, the comparison of the results presented here with the 
ones reported in $[1,2]$ shows that the maximum spread of the hysteresis curve in the TC block is, approximately, three times smaller than that of the steel the block of identical dimensions. From these observations we can conclude that for the amplitude studies of the hysteresis effects of the evolution process it is reasonable to use steel objects, while for studies of the shapes of the hysteresis curves TC objects have advantages.

In order to demonstrate the uniqueness of the hysteresis effect, which is specific for any part of a material object [1, 2, $11]$, the measurement system in this study was moved as a whole to the opposite gauging surface of the block, when already thermistor-1 was closer to the gauging surface. The corresponding hysteresis curve (averaged over several modulation cycles) is shown in Fig. 6. Comparing it the plot of Fig. 5, we conclude that the spans of the curves are, practically the same, but the sign of the effect is changed to the opposite. It means that the maximum value in the plot of Fig. 6 corresponds to minimum value of the plot in Fig. 5, and the minimum value of Fig. 6 corresponds to the maximum value in Fig. 5. The magnitudes of steps (or the variations from the initial position at the last point of the previous period of the modulation cycle to the extreme value reached in the new period) for the cooling and for the heating periods are equal to each other to within a couple of percent. These experiments demonstrate that an initially homogeneous block (made of uniform material) looses the symmetry in its properties, so that the surfaces of the artifact, which are located at the equal distances from the point of the absorption of EM energy and momentum, still radiate different amounts of energy into the surrounding world $[7,8]$. The other conclusion of primary importance is that the periodic process (resulting in the detection of the hysteresis effect) in accordance with the principles of thermodynamics is definitely irreversible in time $[1,2,7]$. Indeed, the studied system is an open one, and the hysteresis curve characterizes the amount of energy, which is lost from the studied part of the system during one modulation cycle and which is then removed by the air-conditioning system $[2,7]$, thus heating the Earth and its atmosphere.

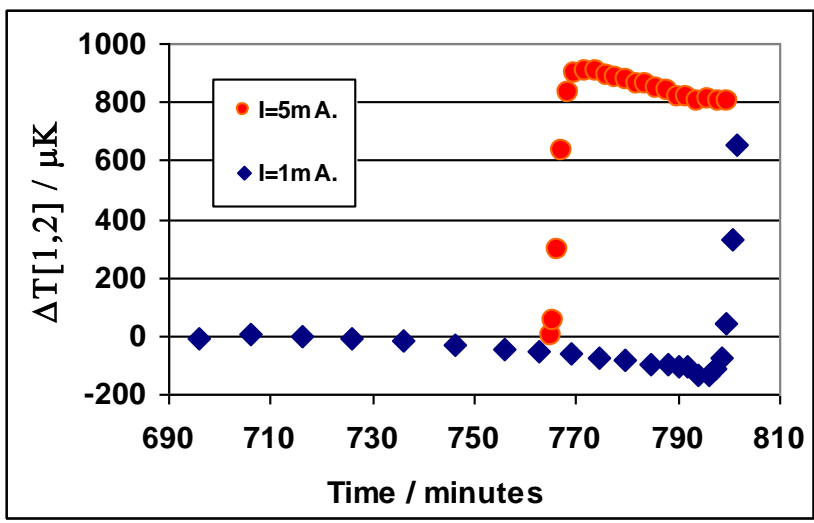

Fig.6. The record of the thermal hysteresis curve, when thermistor-1 was close to the gauging surface of the block, the temperature difference $\mathbf{T}[\mathbf{2 , 1}]$ was $1.17 \mathrm{mK}$, and the distance between the adapters was $13.5 \mathrm{~mm}$. Relative to the plot of Fig. 5, the sign of the effect is changed to the opposite one.

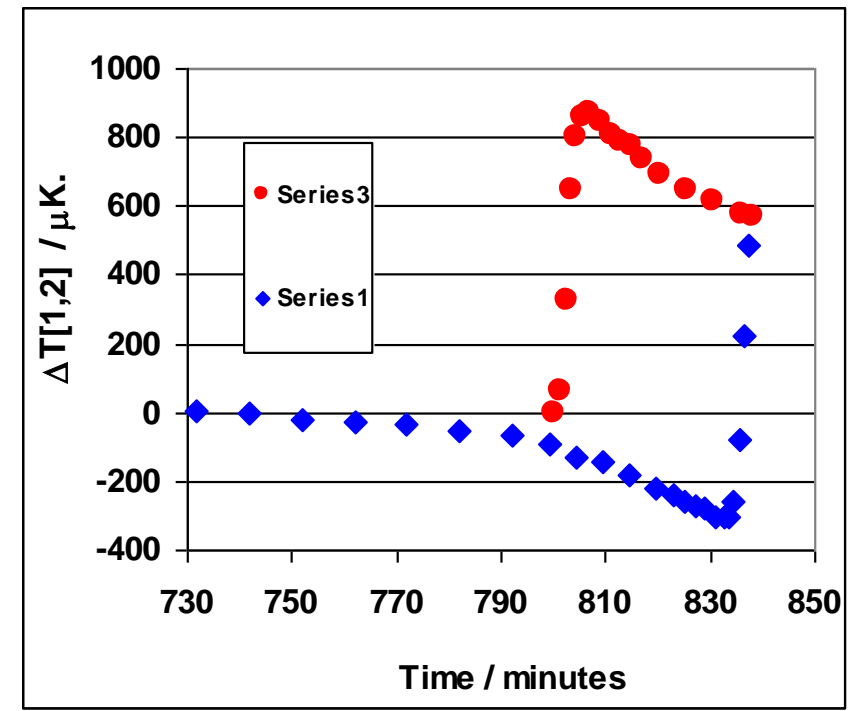

Fig.7. The record of the thermal hysteresis curve, when thermistor-1 was close to the gauging surface of the block, the temperature difference $\mathbf{T}[\mathbf{2 , 1}]$ was $8.14 \mathrm{mK}$, and the distance between the adapters was $13.5 \mathrm{~mm}$.

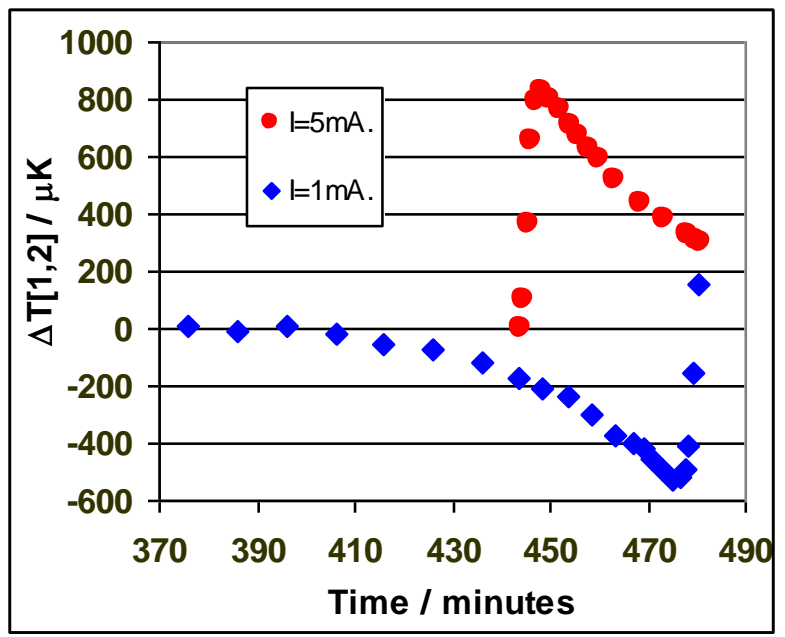

Fig.8. The record of the thermal hysteresis curve, when thermistor-1 was close to the gauging surface of the block, the temperature difference $\mathbf{T}[\mathbf{2 , 1}]$ was $17.2 \mathrm{mK}$, and the distance between the adapters was $13.5 \mathrm{~mm}$.

In order to demonstrate the effect of the external source of EM energy on the evolution process in the TC block, an auxiliary heat source No. 1 (shown in Fig. 1) was switched on. The DC current in this source was adjusted to create a constant heat flux that resulted in the appearance of the temperature difference between the thermistors 2 and 1 of a few $\mathrm{mK}$. Then the modulation of the PRT current was used, and after the stabilization of the mean temperature in the Dewar, the hysteresis curve was recorded. As demonstrated earlier in $[8,11]$, the very presence of the effect of the external heat source results in the variations of the parameters of the hysteresis curve (which is produced as a result of synchronous detection of the periodic modulation of the energy flux produced the PRT). This means that the principle of super-position is not valid for the interaction of electromagnetic fields with matter $[2,11]$. The effect of an auxiliary source of EM radiation on the form of the 


\section{New Studies of Thermal Evolution Process}

hysteresis curve in the TC gauge block is demonstrated by the plots of Figs. 7 and 8. Here, the temperature difference $\mathbf{T}[\mathbf{2}, \mathbf{1}]$, which was determined as a mean value for the reference points at the end of the cooling period, was equal to $8.14 \mathrm{mK}$ for Fig. 7, and $17.2 \mathrm{mK}$ for Fig. 8. From the comparison of the hysteresis curves in Figs. 6-8, it is clear that for the broad-band thermal radiations the violation of the principle of the superposition in the TC block is quite large and can be easily detected by simple eye estimation.

From these plots it is easy to find that the maximum of the quantity $\Delta \mathbf{T}[\mathbf{1 , 2}]$ is dropping significantly with the increase of the temperature difference $\mathbf{T}[\mathbf{2}, \mathbf{1}]$, which arises due to the radiation of the auxiliary heat source. Further, the increase of $\mathbf{T}[\mathbf{2 , 1 ]}$ results in the substantial decrease of the hysteresis effect (i.e. the quantity $\Delta \mathbf{T}[\mathbf{1 , 2}]$ ) at the end of the heating period of the modulation cycle. Besides that, the increase of the temperature difference $\mathbf{T}[\mathbf{2 , 1}]$ reduces the amount of energy dissipated from the system and decreases the time interval for the quantity $\Delta \mathbf{T}[\mathbf{1 , 2}]$ to reach its maximum value.

Thus, the experimental studies performed here and in $[1,2$, $7,8,11]$ (which cover different materials, different durations and forms of the modulation signal and different positions of the measuring system on the surfaces of the artifacts) are presenting the type of experiment that falsifies all the theories, which were developed in accordance with Newtonian paradigm [4]. This statement can be made in accordance and in strict agreement with K. Popper - A. Einstein principle [11], which A. Einstein formulated in a concise form in his historic phrase: "No amount of experimentation can ever prove me right; a single experiment can prove me wrong."

\section{CONCLUSIONS}

First, it should be taken into consideration that the scope of these experimental studies is far beyond possibilities of theoretical physics to give an adequate mathematic description for any natural process, or, in particular, for the thermal evolution process, that is studied here as an example of the simplest and best experimentally investigated process. Indeed, according H. Poincaré results of the year 1887, mathematics is unable to present in the general case the solution for the consecutive states of a rigid, three-body system, in which the bodies are interacting gravitationally without changing their properties in that interaction process. Meanwhile, as it follows from Fig. 1 (even in the description of the hysteresis effect as a component of the evolution process) we need to include at least 8 objects interacting through EM fields. Indeed, in Fig. 1 we see: a block with three thermometers attached to it; the auxiliary heat source located inside the Dewar; the Dewar vessel and the air-conditioning system; the Earth and its atmosphere as the objects accepting the fluxes of the energy and momentum from the studied system. Besides that, as shown also experimentally here, the principle of superposition is not valid in interactions of EM field with matter, and thus all the interacting objects are changing continuously their properties in the process of interaction. The experiments presented here give another confirmation (under new experimental conditions and for another material) to the basic features of the evolution process $[2,11]$ :
1) there is no symmetry in space for material objects interacting with each other through EM field;

2) the hysteresis effect is specific for any part of a material object interacting with the field; it is characterized by the its own specific time delay, by its own intensity, and by its own spectrum of the radiated field (that is related to the temperature of that part of the object);

3) the principle of superposition is fundamentally not valid for EM fields interacting with material objects:

4) the successive states of a object interacting with the material objects of the environment can be presented only as an irreversible in time, non-repeatable process (for which each state is basically unique).

As the principle of superposition is not valid and each part of an object is radiating EM field, each object in the system of many material objects interacting with the EM field does produce some contribution to the evolution processes in all other partners. So, for the material world, we have an interrelation that was discovered by Gottfried Wilhelm Leibniz before 1714. In $\$ 56$ of his famous "La Monadologie" he wrote [13]: "Now this interconnection, or this adapting of all created things to each one, and of each one to all the others, brings it about that each simple substance has relational properties that express all the others, so that each simple substance (monad) is a perpetual living mirror of the Universe, differing according to the different point of view of each monad." And in his fourth letter to Dr. Clarke he added [14]: "The Nature of every simple Substance being such, that its following State is a consequence of the preceding one; here now is the cause of the Harmony found out. It needs only to make a simple Substance become once and from the beginning, a representation of the Universe, according to its Point of view; Since from thence alone it follows, that it will be so perpetually; and that all simple Substances will always have a Harmony among themselves, because they always represent the same Universe."

In agreement with the fundamental principles of G. W. Leibniz, it is concluded in [11] from the results of experimental studies that all the processes on Earth are irreversible in time and non-repeatable. And this is just a consequence of the fact that the quantity of the solar energy (received at any part of the Earth surface) can be characterized only as a non-repeatable irreversible process, for which the description in terms of mathematics is not possible. (Indeed, it is known from astronomical observations that the rotation of the Earth is decelerating relative to the stars, and that the rotational process is not expressible in terms of mathematics [11]. Besides, the Earth itself cannot be considered as a rigid body).

Here, it is worth reminding that at the end of his scientific activity, Albert Einstein was, probably, deeply dissatisfied with some of his earlier works and scientific statements, and he sent a very emotional letter of 22.12.1950 to E. Schrödinger. It this letter A. Einstein wrote [15]: "It is quite hard to accept that we still are in the stage of babies in their diapers, and it is not surprising that the fellows are unwilling to admit this (even to themselves)." 
Extremely important were the philosophical views of Wolfgang Pauli of that period. W. Pauli was publicly giving openly support to Einstein's position during his last period of scientific activity. It was W. Pauli who was able actually to re-discover the main principles of G. W. Leibniz philosophy. For example, W. Pauli wrote in his letter of 1951 (to M. Fierz): "That which is physically unique cannot be separated from the observer anymore - and therefore falls through the net of physics. The individual case is occasio and not causa. I am inclined to see in this "occasio" - which includes the observer and his choice of the experimental setup and procedure - a "revenue" of the "anima mundi". La donna é mobile - also the anima mundi and the occasio" [15]. In this respect, quite remarkable and impressive are the statements of the philosopher Charles Sanders Peirce and the prominent physicist Richard Feynman. C. S. Peirce wrote in his book (published in the year 1891): "Now the only possible way of accounting for the laws of nature and for uniformity in general is to suppose them results of evolution," [4b]. And many years after A. Einstein's death, R. Feynman admitted at one of his interviews: "The only field which has not admitted any evolutionary question is physics. Here are the laws, we say...but how did they get that way, in time? So, it might turn out that they are not same all the time and that there is a historical, evolutionary question," [4c].

\section{ACKNOWLEDGMENT}

The author gratefully acknowledges the technical and moral support of our experimental studies by the staff of the National Metrology Institute of Brazil (INMETRO). The author is grateful to the staff of the Physics Department of the Yeditepe University (Turkey) for useful discussions and for the offered possibility to present the pioneering results in the field of thermal evolution processes at the International Conference "Chaos-2013" that formed the basis of the set of the following publications in the field.

\section{REFERENCES}

[1] A. Titov, I. Malinovsky, "Experimental demonstration of time-irreversible, self-ordering evolution processes in macroscopic quantum systems" in "Chaotic Modeling and Simulation" (CMSIM), V.1, pp. 79-93, 2014.

[2] A. Titov, "First observations of thermal surface energy and of thermal evolution process." , Journal of Science Frontier Global Research, (GJSFR), V. 15, Issue 3, Version 1.0, pp.1-49, 2015.

[3] R. Loudon, L. Allen and D. F. Nelson, "Propagation of electromagnetic energy and momentum through an absorbing dielectric.", Phys. Rev. E, vol. 55, pp. 1071-1085, 1997.

[4] L. Smolin, "Time reborn." , Houghton Mufflin Harcourt, Boston New York, 2013, pp. 114-174 [4a]; p. XXV [4b]; p. XXVI [4c].

[5] K. Gödel, "On formally undecidable proporsitions of Principia Mathematica and related systems I", 1931, in S. Feferman, (Ed), "Kurt Gödel Collected works", V. 1, Oxford University Press, pp. 144-195, 1986

[6] J. D. Jackson, "Classical Electrodynamics.", J. Willey and Sons, 3-ed, p. 14, 1999.

[7] A. Titov, I. Malinovsky, "Demonstration of the existence of thermal surface energy and its impacts." European Scientific Journal, V.9, No. 24, pp. 11-44, 2013.

[8] A. Titov, "Thermal Evolution Process and its Relation to Some Philosophical Problems of Physics.", Proceedings of the 2nd International Conference on Fluid Flow, Heat and Mass Transfer, pp.153-1 - 153-9, Ottawa, Ontario, Canada, 2015.

[9] A.Titov, I. Malinovsky, "Nanometrology and high-precision temperature measurements under varying in time temperature conditions." Proc. SPIE, V. 5879, "Recent Developments in
Traceable Dimensional Metrology”, J. E. Decker; Gwo-Sheng Peng, (Eds.). SPIE, Belingham, WA, 2005; pp. 587902-1 - 587902-10. DOI: $10.1117 / 12.616630$.

[10] A.Titov, I. Malinovsky, M. Erin, H. Belaidi, R. S. Franca, "Precise certification of the temperature measuring system of the original Kosters interferometer." , Proc. SPIE, V. 5879, "Recent Developments in Traceable Dimensional Metrology”, J. E. Decker; Gwo-Sheng Peng, (Eds.), Belingham, WA, 2005; pp. 587902-1 587902-10. DOI: 10.1117/12.616662.11.

[11] A. Titov," Experimental Demonstration of the Relational Character of Time in Natural Processes; Concept of Time in the Relational World", World Journal of Research and Review (WJRR), ISSN: 2455-3956. V. 5, Issue. 4, pp. 70-99, 2017. DOI: 10.31871/WJRR.5.4.9

[12] J. A. Ewing, "On effects of retentiveness in the magnetization of iron and steel", in "Proceedings of the Royal Society of London", V. 34, p. 39, 1882.

[13] G. W. Leibniz, "La Monadologie", 1714; R. Latta, (trans.), Oxford: Clarendon Press, 1898; eBooks@ Adelaide, University of Adelaide, South Australia, 2014.

[14] H.G. Alexander, Ed, "The Leibniz-Clarke Correspondence." Manchester: Manchester University Press, 1956.

[15] A. Zeilinger, "On the Interpretation and Philosophical Foundation of Quantum Mechanics"” in: "Vastakohtien todellisuus". U. Ketvel et al. (Eds.), Helsinki University Press, 1996. 